Bulletin d'études orientales

\title{
Aux sources de la métrique quantitative ('arūẹ) kurde
}

\section{Amr Taher Ahmed}

\section{(2) OpenEdition}

\section{Journals}

Édition électronique

URL : http://journals.openedition.org/beo/4939

DOI : 10.4000/beo.4939

ISSN : 2077-4079

Éditeur

Presses de l'Institut français du Proche-Orient

Édition imprimée

Date de publication : 1 avril 2017

Pagination : 157-176

ISBN : 978-2-35159-731-6

ISSN : 0253-1623

Référence électronique

Amr Taher Ahmed, « Aux sources de la métrique quantitative ('arūd) kurde », Bulletin d'études orientales [En ligne], LXV | 2017, mis en ligne le 01 avril 2019, consulté le 06 janvier 2021. URL : http:// journals.openedition.org/beo/4939; DOl : https://doi.org/10.4000/beo.4939 


\title{
Aux sources de la métrique quantitative ('arūọ) kurde
}

\author{
Amr Taher AHMED
}

Résumé : La métrique quantitative de la poésie kurde trouve ses racines dans l'héritage halîlien et la métrique arabe. En pratique, cependant, c'est à la poésie persane que les poètes kurdes classiques font le plus souvent référence. La question qui se pose est dès lors la suivante: l'adoption du 'arū ḍ en kurde s'est-elle opérée par contact direct avec la tradition arabe ou par l'intermédiaire de la poésie persane? Les récentes avancées réalisées dans l'étude de la métrique des trois langues nous fournissent désormais les instruments techniques et statistiques nécessaires pour répondre de façon concluante. Fort de ces acquis, le présent article analyse les mètres et constituants métriques (pieds et variantes) en usage dans la poésie kurde classique. On établit ainsi qu'inscrite dans le sillage de la poésie persane, la métrique kurde quantitative présente un cas d'adoption du système halīlien au second degré.

Mots clés : 'arūd, métrique quantitative, poésie kurde, poésie persane, poésie arabe.

\begin{abstract}
The metrical system of quantitative Kurdish poetry finds its origins in the halilian tradition of Arabic prosody. In practice, however, classical Kurdish poets rather referred to Persian poetry instead. The question, therefore, is the following: Was 'arüd adopted in Kurdish by direct contact with the Arabic tradition, or was it brought about through the medium of Persian poetry? The recent progress in the study of the prosody of all three languages now provides the technical and statistical knowledge necessary to answer concludingly. Based on the latest relevant findings, this article analyses the meters and metrical constituents (feet and variables) in use in classical Kurdish poetry. As a consequence, it can be claimed that quantitative Kurdish metrics developed in the wake of Persian poetry, thus presenting a case of adoption of the halilian system in the second degree.
\end{abstract}

Keywords: 'arūd, quantitative metrics, Kurdish poetry, Persian poetry, Arabic poetry.

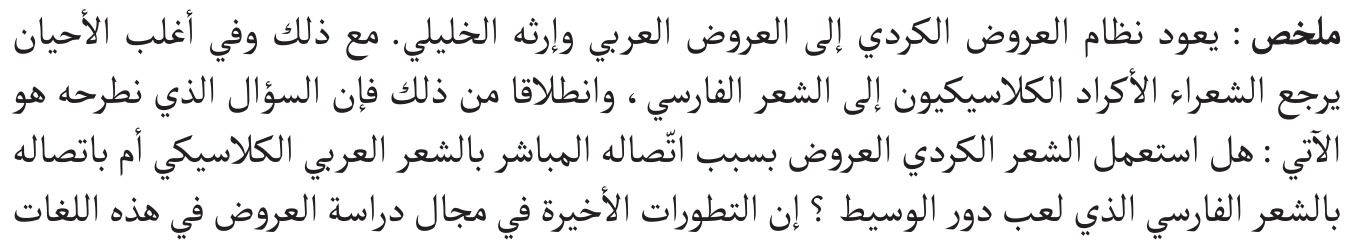




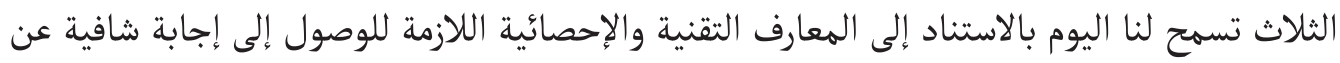

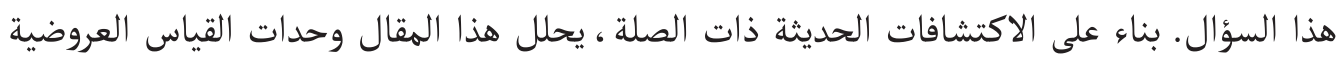

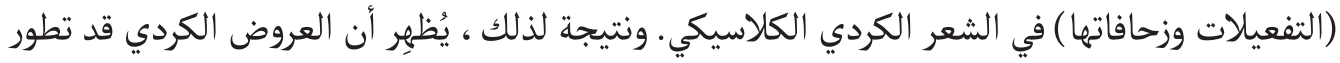

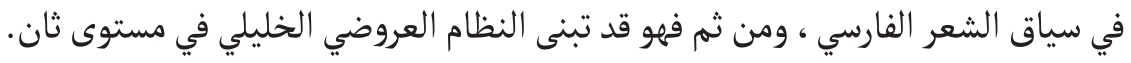

الكلمات المحوريّة : العربية ، فونولوجيا ، فونولوجيا توليدية ، إيقاع ، تمثيلات معجمية ، فونولوجيا تقريرية ، قيود. 


\section{Introduction}

Aux environs de l'an 1682, Ehmed Xanî ${ }^{1}$ (1650-1707), poète kurde réputé et auteur du célèbre masnawī d'amour Mem û Zîn (Mam et Zīn), achevait son Nûbara biçûkan (Primeur pour les enfants) ${ }^{2}$, manuel dédié à l'apprentissage du vocabulaire arabe et de la métrique. Ayant rendu les grâces d'usage (hamd et șalawāt) au Créateur et à son Prophète, l'auteur précise en préambule la fonction qu'il réserve à son ouvrage. Le manuel, explique-t-il, est destiné aux jeunes élèves des écoles traditionnelles, maktab ou madrasa, qui auront déjà achevé une première lecture intégrale du Coran, sans encore en comprendre le sens. L'ouvrage leur tiendra lieu de guide dans leur initiation à la philologie, en sorte qu'ils pourront saisir «sans difficulté » le sens des textes auxquels ils seront confrontés ${ }^{3}$.

Conçu en forme de lexique bilingue, le Nûbar offre une structure claire. Sur quelques 180 vers mesurés et rimés, répartis en douze sections appelées qit ${ }^{c} a$, Xanî juxtapose des mots arabes et leur synonyme kurde. Pourtant, l'identité des deux langues n'est jamais explicitée. Le nom des langues arabe et kurde n'apparaît ni dans le titre de l'ouvrage, ni dans le glossaire; en introduction, le poète se contente d'annoncer « quelques termes des langues » (Ef çend kelîme ji luxatan $)^{4}$.

La nature prosodique de l'ouvrage, en revanche, est clairement identifiée. Elle s'annonce à l'ouverture de chaque section par un double signal. Le premier est contenu dans le titre, qui énonce, en arabe, le nom complet du mètre employé dans la section. Ainsi, le premier qiț $a$ du lexique s'ouvre sur la mention: "Section [fondée] sur le mètre rağaz mațwì maḩbūn » (Al-qiț'a fì baḥr al-rağaz al-mațī al-maḩbūn). Le second signal apparaît dans le deuxième vers de chaque section. Après un premier vers introductif, le deuxième vers se compose en effet de la formule générale du mètre employé (premier hémistiche) et du nom, complet ou partiel, du modèle de vers correspondant (second hémistiche). Ainsi par exemple, le deuxième vers de la première section du lexique se lit :

Muftacilun mufä́cilun muftacilun mufä́cilun

Behrê recez ku xebn û tey ger bibitin rewan e xweş

muftacilun mufä́cilun mufta'ilun mufäcilun

Le mètre rağaz devient fluide [et] agréable s'il est [affecté] du habn et du tayy. ${ }^{5}$

C'est d'ailleurs la métrique qui permet de comprendre que le premier manuel de prosodie kurde soit apparu en kurmānǧì, plutôt que dans tout autre parler du kurde. Il s'agit là, selon toute évidence, d'un phénomène historique. Certes, il est de coutume de rapporter les débuts de la littérature kurde aux textes sacrés des Yārsāns, courant religieux ${ }^{6}$ à l'origine d'une littérature en gorānī que l'on fait habituellement remonter

1. Tous les termes kurdes sont transcrits en caractères latins conformément aux usages de transcription dans cette langue.

2. XEZNEDAR 2002, p. 373.

3. XANî, Nûbar, p. 7-8.

4. Ibid.

5. Ibid., p. 11. Pour la définition du habn et du tayy, voir PAOLI 2008, p. 105-106.

6. Sur les Yārsāns, voir VALI 2011. 
au $\mathrm{X}^{\mathrm{e}}$ siècle de l'ère commune, voire plus haut ${ }^{7}$. En l'absence de manuscrits anciens, cependant, ces datations demeurent hypothétiques ${ }^{8}$. Toujours est-il que ces poèmes sacrés se distinguent de l'ensemble de la poésie kurde classique par un trait prosodique notable. En effet, la poésie des Yārsāns est exclusivement fondée sur une métrique syllabique, qui tire ses origines de la poésie kurde populaire. Ce n'est qu'avec l'émergence de la littérature kurmānğì au XVI ${ }^{\mathrm{e}}$ siècle que l'on assiste à l'introduction de la métrique quantitative arabe ('arūẹ) dans la langue kurde. Jusqu'à l'époque moderne, au demeurant, les poètes kurmānğs ont rarement eu recours aux mètres syllabiques. Il en allait de même dans la poésie kurde sorānī, née au début du XIX ${ }^{\mathrm{e}}$ siècle, jusqu'à ce qu'au milieu du $\mathrm{XX}^{\mathrm{e}}$ siècle, le poète 'Ebdulla Goran (1904-1962) et une poignée de réformateurs ne contestent l'usage du 'arūḍ ${ }^{9}$. C'est ainsi que la métrique quantitative fut progressivement bannie de la poésie kurde moderne au profit de la métrique syllabique ${ }^{10}$.

Il serait vain de chercher dans le manuel de Xanî une quelconque allusion aux origines de la métrique quantitative kurde. À cet égard, l'absence d'un véritable traité de métrique kurde sur toute la période classique apparaît significative. Contrairement aux Arabes, mais aussi aux Persans, les Kurdes ne se sont guère souciés d'élaborer à ce sujet des ouvrages théoriques. Aussi ne trouve-t-on pas de littérature réservée à la définition de la métrique quantitative, à la classification des mètres, à l'expression des règles propres au 'arū ou a fortiori aux relations qu'entretient celui-ci avec ses équivalents arabe et persan ${ }^{11}$.

Il faut attendre l'ère moderne pour assister à l'efflorescence d'une série d'ouvrages consacrés, en tout ou partie, à l'étude du 'arūd kurde ${ }^{12}$. À la question de savoir d'où il procède et quelles en sont les origines, les métriciens kurdes apportent toutefois une réponse unanime et sans ambiguité : la métrique kurde quantitative trouve sa source en arabe, dans l'héritage halilien. En revanche, rares sont ceux qui s'aventurent sur le terrain du comparatisme pour aborder cette autre question, d'ordre historique : l'adoption en kurde de ce système métrique s'est-elle opérée directement, au contact de la poésie arabe, ou y a-t-il eu un intermédiaire, dans la figure de la poésie persane ? La question prend tout son sens si l'on songe que, dans leurs créations poétiques, tant en kurmānǧī qu'en sorānī, les Kurdes se sont largement inspirés des modèles poétiques arabe et persan à travers l'histoire, et que, pour des raisons qu'il serait trop long de détailler ici, le modèle persan fut de tous temps prépondérant ${ }^{13}$.

Ironie du sort, c'est à son principal adversaire, Goran, qu'il échut de formuler un premier avis sur l'adoption du 'arūd en kurde. Cette réflexion se rencontre dans des notes

7. XEZNEDAR 2001, p. 224-306 ; BOREKEYî 2008, p. 47-121.

8. VALi 2012, p. 58.

9. Sur ces questions, voir GORAN 1980, p. 3-4 ; BîMAR 1970, p. 2-3 ; GORAN 2002, p. 157-158.

10. AHMED 2012, p. 70-79.

11. Parmi les nombreux traités de métrique classiques arabes et persans, signalons ces deux ouvrages de référence :

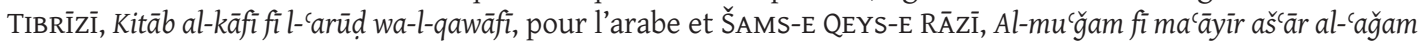
pour le persan.

12. Voir XEZNEDAR 1962 ; BÎMAR 1992 ; GERDî 1999 ; HARDî 2004 ; HEMEXAN 2004.

13. 'ARIF 1974. 
de cours, rédigées vers la fin de sa vie lorsqu'il enseignait la langue et la littérature kurdes à la faculté des lettres de l'Université de Bagdad, et publiées depuis ${ }^{14}$. Raisonnant sur la production poétique kurde de son temps, et sur les options métriques qu'elle incarne, Goran identifie dans ces pages deux tendances opposées : la voie classique et la voie moderne. Si la seconde s'illustre par un retour militant à la poésie populaire et par le choix de la métrique syllabique, la première s'inscrit dans le sillage des «poètes [kurdes] classiques qui ont adopté, il y a de cela bien longtemps, les mètres arabes 'arūdì par le truchement de la poésie persane ${ }^{15} »$.

Énoncée comme une évidence, l'affirmation de Goran n'est alors étayée par aucune analyse. De tous les métriciens kurdes, 'Ezîz Gerdî est le seul à avoir interrogé ce postulat. L'ouvrage qu'il consacre à la métrique kurde a paru en 1999. Gerdî y examine en parallèle la relation qu'entretient le 'arüḍ kurde avec les traditions métriques arabe et persane. Sans appuyer sa démonstration sur une comparaison chiffrée, il conclut néanmoins à juste titre que c'est la poésie persane qui a fourni aux poètes kurdes leur principal modèle métrique ${ }^{16}$.

Inspiré des travaux de Gerdî et fondé sur les derniers progrès réalisés dans les études métriques, le présent travail a pour objet de résoudre les questions qui subsistent quant à la nature exacte des relations entre métriques quantitatives kurde et persane. Les progrès accomplis dans la connaissance de la métrique des trois langues - arabe, persan et kurde nous fournissent désormais les arguments techniques et statistiques nécessaires pour établir que l'assimilation de l'héritage halīlien s'est effectuée en kurde par l'intermédiaire de la poésie persane. Pareille réflexion n'ayant à ce jour inspiré de travaux qu'à quelques rares spécialistes kurdes, il s'agit d'une première tentative du genre dans une langue occidentale. Nous débutons notre étude par l'examen de la plus grande unité métrique, le mètre, avant de nous pencher sur son élément constitutif, le pied.

\section{Le choix des mètres}

Pour des raisons historiques qui dépassent le cadre de notre analyse, la littérature kurde classique compte un nombre de poètes et de poésies très inférieur à ceux des littératures arabe et persane. Les dimensions modestes de ce corpus ont permis à 'Ezîz Gerdî d'établir, dans un ouvrage plus récent, des statistiques précises concernant les mètres et les modèles de vers en usage dans la poésie kurde. Son « Guide métrique de la poésie kurde classique » (Raberî kêşî şếrî kilasîkî kurdî) considère l'œuvre de quatre-vingt-deux poètes ayant composé sinon la totalité, du moins une part importante de leur poésie, selon les règles traditionnelles du 'arū Pour chaque poète, Gerdî établit le décompte des mètres et des modèles de vers usités. Il

\footnotetext{
14. Après le décès de Goran, ses notes de cours furent publiées dans la revue Jîn (Vie), puis rééditées dans GORAN 2002, p. 134-164.

15. Ibid., p. 157.

16. GERDî 1999, p. 292-295.
} 
fournit ainsi des statistiques détaillées de fréquence, avant de conclure son « Guide » par un ensemble de tableaux récapitulatifs ${ }^{17}$.

Si les travaux de Gerdî ont apporté en leur temps une base solide à la connaissance de la métrique kurde, ses statistiques n'en sont pas moins frappées d'un certain déséquilibre. En effet, le chercheur fait fonds sur un corpus composé prioritairement de poètes kurdes de parler sorānī. Sur quatre-vingt-deux poètes recensés, on ne trouve que six poètes kurmānğs. Or, ce déséquilibre était involontaire de la part du savant. Il reflétait seulement l'état de l'édition à l'heure où il établissait ses statistiques: Gerdî avait bien pris en considération toute la poésie kurmānğĩ éditée. Depuis, le corpus d'auteurs disponibles s'est sensiblement élargi. Les œuvres d'une trentaine de poètes classiques de parler kurmānğī ont été découvertes et publiées ${ }^{18}$. Afin d'actualiser les statistiques de Gerdî et de rééquilibrer ses conclusions, nous avons procédé nous-même à la recension métrique de ce nouveau corpus kurmānğì. Ces nouvelles données nous ont permis de compléter les statistiques de Gerdî, auxquelles nous les avons ajoutées. Nous en résumons les résultats finaux dans le tableau I ci-dessous.

\begin{tabular}{|c|c|c|c|c|c|c|c|c|}
\hline mètres & $\begin{array}{c}\text { nb. de } \\
\text { poèmes }\end{array}$ & $\%$ & $\begin{array}{l}\text { octopartite } \\
\text { mutamman }\end{array}$ & $\%$ & $\begin{array}{l}\text { sexpartite } \\
\text { musaddas }\end{array}$ & $\%$ & $\begin{array}{c}\text { quadripartite } \\
\text { murabbac }\end{array}$ & $\%$ \\
\hline hazağ & 4278 & 46,9 & 2863 & 66,9 & 1218 & 28,4 & 197 & 4,6 \\
\hline ramal & 3479 & 38,2 & 2980 & 85,6 & 429 & 12,3 & 70 & 2 \\
\hline mudāāic & 468 & 5,1 & 451 & 96,3 & - & - & 17 & 3,6 \\
\hline rağaz & 407 & 4,4 & 208 & 51,1 & 5 & 1,2 & 194 & 47,6 \\
\hline mutaqārib & 149 & 1,6 & 149 & 100 & - & - & - & - \\
\hline hafif & 139 & 1,5 & - & - & 139 & 100 & - & - \\
\hline muğtatt & 98 & 1 & 98 & 100 & - & - & - & - \\
\hline basit & 33 & 0,3 & 24 & 72,7 & - & - & 9 & 27,2 \\
\hline $\operatorname{sari}^{\mathrm{c}}$ & 30 & 0,3 & - & - & 30 & 100 & - & - \\
\hline munsarih & 10 & 0,1 & 10 & 100 & - & - & - & - \\
\hline mutadārak & 5 & 0,05 & 5 & 100 & - & - & - & - \\
\hline kāmil & 4 & 0,04 & 4 & 100 & - & - & - & - \\
\hline madìd & 2 & 0,02 & 1 & 50 & - & - & 1 & 50 \\
\hline mustațil & 2 & 0,02 & 2 & 100 & - & - & - & - \\
\hline tawil & 1 & 0,01 & 1 & 100 & - & - & - & - \\
\hline Total & 9105 & 100 & 6796 & 74,6 & 1821 & 19,9 & 488 & 5,3 \\
\hline
\end{tabular}

Tableau I

17. GERDî 2003, p. 762-786.

18. Il s'agit des poètes suivants : Cegerxwîn, Dahî (Mela Nezîr Bedewî), Dilhezîn Findikî, Fethî (Mela 'Ebdulfettah Hezrûyî), Hadî (Mela Muhemmed Sibxetullah Liceyî), Haris Bedlîsî, Hesbî (Şêx Hesîb Axtepî), Muhemmed Kerbelayî, Laxerî (Mela 'Umer Tînîsî Muksî), Mela 'Ebdirrehman Melakendî, Mela Ehmed Heyderî, Mela Es'ed Çoqreşî, Mela Ibrahîm Çoqreşî, Mela Ismetullah Karazî, Mela Xelîl Mişextî, Mela Zahir Tendûrekî, Muftî Mela Mehfûz, Muhemed Hadî Qewmanî, Remezan Cizîrî, Rûhî (Şêx Evdirehman Axtepî), Selîm Hîzanî, Serdayî (Selaheddîn Hecî 'Ebdila Wanî), şêx 'Ebdilqadir Hezanî, şêx Muhemmed Emîn Heyderî, şêx Muhemmed 'Ubeydullah Şewqî Efendî, Şêx Qedrî Cizîrî, Şêx Yehya Çoqreşî, Siyahpoş, Wedâî, Xanî (Nûbar). Certains des ouvrages consultés rassemblent les œuvres de plusieurs poètes. 
Notre tableau résume le nombre de poèmes composés sur chaque mètre, chacun étant affecté d'un pourcentage relatif au nombre total de poèmes. Les statistiques sont affinées de manière à prendre en compte les trois catégories de vers envisageables pour chaque mètre : octopartite, sexpartite et quadripartite. Ainsi, on recense par exemple 4278 poèmes composés sur le mètre hazağ, chiffre qui correspond à $46,9 \%$ du corpus total examiné. Sur ces 4278 occurrences, $2863(66,9 \%)$ sont des octopartites, 1218 (28,4\%) des sexpartites et 197 (4,6\%) seulement des quadripartites.

Le corpus poétique persan est à la fois trop abondant et trop complexe pour que l'on puisse envisager de lui appliquer une étude similaire. Aussi, en dépit de la modernisation des formes poétiques engagée à l'orée $\mathrm{du} \mathrm{Xx}^{\mathrm{e}}$ siècle et du développement du vers libre, la métrique quantitative conserve une place considérable dans la poésie contemporaine. $\mathrm{Ce}$ phénomène, associé à l'extension historique et géographique qu'a pu connaître la langue persane, voue d'avance à l'échec toute velléité de recension exhaustive des usages du 'arūd persan. Ce constat n'a pas empêché certains spécialistes de conduire des recherches de tendances qui, pour approximatives qu'elles soient, ne s'en révèlent pas moins pertinentes. De fait, les résultats obtenus par ces études mettent au jour un ensemble de récurrences et de régularités qui témoignent d'une remarquable continuité dans l'usage de la métrique quantitative persane à travers son histoire.

Il existe à ce jour deux études de ce genre. La première, publiée par Elwell-Sutton en 1976, est de vaste ambition puisqu'elle s'appuie sur un large échantillon de textes choisis "au hasard», toutes périodes confondues, depuis les premiers vers persans recensés jusqu'au vers libre non inclus ${ }^{19}$. Dans un corpus constitué de 20155 occurrences métriques, Elwell-Sutton dénombre 87 modèles de vers. Plus récemment, on a pu examiner les points de divergence et de convergence entre les usages métriques persans et arabes, sur un corpus limité aux "premiers poètes persans » des $\mathrm{IX}^{\mathrm{e}}$ et $\mathrm{X}^{\mathrm{e}}$ siècles ${ }^{20}$. Le tableau II (ci-dessous) reproduit les résultats d'Elwell-Sutton, plus représentatifs sur la durée ${ }^{21}$.

19. L'auteur affirme en outre que les statistiques établies sur l'œuvre individuelle d'un poète ou sur l'ensemble de la production d'une période donnée corroborent les résultats de son étude avec une « constance remarquable ». Voir ELWELL-SUTTON 1976, p. 145.

20. AHMED et PAOLi 2010, p. 135-136.

21. Précisons que le tableau II que nous présentons ici ne se rencontre pas à l'identique chez Elwell-Sutton; il procède d'une reconstitution pour les besoins du présent article. De fait, voulant faire l'économie de la lourde terminologie métrique traditionnelle, Elwell-Sutton propose dans son ouvrage un classement alternatif des mètres persans, inspiré par les travaux du philologue iranien Hānlarī, et au final, également compliqué. Les 208 occurrences de vers persans identifiées (dont 39 inclassables selon la métrique classique) sont réparties en quatorze groupes, selon des critères croisant identification des pieds constitutifs (trisyllabe composé d'une brève et de deux longues, quadrisyllabe composé d'une brève et de trois longues, quadrisyllabe composé de deux brèves et de deux longues, etc.), disposition des pieds dans l'hémistiche (répétitive, comme dans $\cup--/ \cup--/ \cup--$ ou alternative, comme dans $-\cup \cup-/-\cup-\cup-/-\cup \cup-$ ) et nombre total de syllabes par hémistiche. Chaque modèle se voit affecté d'un code correspondant à ces trois critères. Ainsi par exemple, la configuration $(\cup--/ \cup--/ \cup--)$ est identifiée comme 1.1.9, ce qui signifie que le modèle de vers appartient au groupe 1 (pieds trisyllabes avec une brève et deux longues), qu'il se constitue du premier des trois types de pieds du groupe $\left(\cup_{-}\right)$et qu'il contient neuf syllabes par hémistiche. C'est également selon ce système que sont rapportées les données statistiques et les tableaux récapitulatifs. Les 87 modèles de vers référencés dans ces tableaux devaient donc être reconvertis à la nomenclature métrique classique (ramal, hazağ, etc.) pour se prêter à la comparaison avec les données statistiques kurdes. La reconversion des données d'Elwell-Sutton 


\begin{tabular}{|c|c|c|c|c|c|c|c|c|}
\hline mètres & $\begin{array}{l}\mathrm{nb} . \mathrm{de} \\
\text { poèmes }\end{array}$ & $\%$ & $\begin{array}{l}\text { octopartite } \\
\text { mutamman }\end{array}$ & $\%$ & $\begin{array}{l}\text { sexpartite } \\
\text { musaddas }\end{array}$ & $\%$ & $\begin{array}{c}\text { quadripartite } \\
\text { murabbac }^{c}\end{array}$ & $\%$ \\
\hline ramal & 5543 & 27,5 & 4663 & 84,2 & 880 & 15,87 & - & - \\
\hline hazağ & 4465 & 22,1 & 2681 & 60,04 & 1783 & 39,93 & 1 & 0,02 \\
\hline muğtatt & 3181 & 15,8 & 3181 & 100 & - & - & - & - \\
\hline muḍ̄āic & 3159 & 15,7 & 3084 & 97,62 & 75 & 2,37 & - & - \\
\hline hafif & 1799 & 8,9 & 8 & 0,44 & 1791 & 99,55 & - & - \\
\hline mutaqārib & 644 & 3,2 & 642 & 99,68 & 2 & 0,31 & - & - \\
\hline munsarih & 556 & 2,7 & 547 & 98,38 & 7 & 1,25 & 2 & 0,35 \\
\hline rağaz & 435 & 2,1 & 432 & 99,31 & 3 & 0,68 & - & - \\
\hline $\operatorname{sarīi}^{c}$ & 222 & 1,1 & - & - & 222 & 100 & - & - \\
\hline qarīb & 58 & 0,2 & - & - & 58 & 100 & - & - \\
\hline mušākil & 20 & 0,09 & 20 & 100 & - & - & - & - \\
\hline muqtadab & 15 & 0,07 & 15 & 100 & - & - & - & - \\
\hline basit & 9 & 0,04 & 9 & 100 & - & - & - & - \\
\hline mutadārak & 7 & 0,03 & 1 & 14,28 & - & - & 6 & 85,71 \\
\hline madid & 1 & 0,005 & 1 & 100 & - & - & - & - \\
\hline Total & 20114 & 99,535 & 15284 & 75,98 & 4821 & 23,96 & 9 & 0,04 \\
\hline
\end{tabular}

Tableau II

Les analyses développées dans les pages qui suivent s'appuient largement sur les résultats présentés dans ces tableaux. Ils serviront d'outil de comparaison pour déterminer lequel des deux systèmes métriques, arabe ou persan, marque la plus grande proximité avec les usages relevés en kurde.

\section{Mètres inusités}

Sur les seize mètres définis par la théorie halīlienne, certains sont amplement représentés dans la poésie arabe, d'autres très peu. Le phénomène relève de la « surgénération » métrique inhérente au système élaboré par al-Halîl, comme l'ont très bien montré Bohas et Paoli ${ }^{22}$ : animée d'un souci de symétrie peu représentatif de l'usage, la théorie génère des modèles de mètres qui ne correspondent pas à la pratique réelle des

a notamment fait apparaître un reste: sur l'ensemble des occurrences recensées, 21 ne correspondent à aucun mètre classique et 19 pourraient être rangées sous deux mètres différents. Par souci d'exactitude, nous avons exclu ces 40 occurrences de notre tableau. Nous avons en outre relevé une erreur de détail dans les calculs de l'auteur: le tableau des modèles du groupe 4 d'Elwell-Sutton totalise 8799 occurrences au lieu de 8 800. Par conséquent, notre tableau II retranche au total 41 occurrences au corpus d'Elwell-Sutton (nous en présentons 20114 au lieu de 20 155). Ceci étant, cette différence n'affecte guère les résultats d'ensemble. Voir ELWELL-SUTTON 1976, p. 83-167 ; HĀNLARĪ 2007, p. 165-216.

22. BOHAS et PAOLI 1997, p. 152-153. 
poètes et ne sont pas attestés dans les textes. Ainsi le munsarid, pur produit de la théorie des cercles, est demeuré quasiment ignoré des poètes. On le dit, pour cette raison, muhmal (inusité) ${ }^{23}$.

La métrique halīlienne s'est imposée dans la poésie persane dès les VIII ${ }^{\mathrm{e}}$-IX ${ }^{\mathrm{e}}$ siècles. Certes, elle ne s'était pas transmise sans changement : il lui avait fallu s'adapter à la langue indo-européenne, et sans doute aux formes poétiques existantes, au prix de quelques aménagements des règles et des usages. À leur tour, certains mètres se sont vus privilégiés par les poètes, au détriment d'autres, inusités. De ce point de vue, la poésie persane marque des différences notables avec la tradition arabe, et les mètres muhmal, de part et d'autre, sont bien distincts.

En arabe, quatre mètres se partagent à eux seuls près de 80 \% de la production poétique traditionnelle : le țawil, le basiț, le wāfir et le kāmil ${ }^{24}$. Or, au XIII ${ }^{\mathrm{e}}$ siècle déjà, le métricien iranien Šams-e Qeys comptait ces derniers au nombre des mètres les moins usités parmi les Persans, aux côtés d'un cinquième, le madìd. S'il se trouvait qu'un Persan en fît usage, ajoute encore le métricien, ce serait, comble d'artifice et pure imitation des Arabes, au seul souci d'exhiber son habileté poétique ${ }^{25}$. Les statistiques établies pour la métrique persane confirment en tout point l'affirmation de Šams-e Qeys. Sur les 841 occurrences métriques examinées chez les premiers poètes persans, ces cinq mètres ne sont représentés, en tout et pour tout, que par une unique occurrence de tawīl attribuée à Rūdakī ${ }^{26}$. Les statistiques d'Elwell-Sutton (tableau II) le confirment: aucune occurrence de kāmil ni de tawïl n'est attestée. On ne recense qu'une unique occurrence de madìd et neuf de basiț. À eux deux, ces mètres totalisent péniblement $0,045 \%$ du corpus étudié.

Une tendance similaire se dégage du tableau I, qui recense les usages de la métrique kurde: le kāmil et le tawīl comptent parmi les moins fréquents des quinze mètres en usage. Ensemble, ils représentent un total peu significatif de 0,05\% de la poésie kurde traditionnelle. Le basit, pour sa part, ne dépasse pas le seuil des $0,3 \%$ des poèmes. Ainsi, ces trois fondamentaux de la poésie arabe s'arrogent au total à peine 0,35\% du corpus kurde. Comme en persan, le madìd enregistre également, avec seulement deux occurrences, un taux d'usage très faible en kurde : 0,02 \%. Si l'on admet avec Nașīr al-Dīn Ṭūsî, auteur, au $\mathrm{XIII}^{\mathrm{e}}$ siècle également, d'un autre manuel de référence, que le recours aux mètres rares chez les Persans est indice de "préciosité » (takallof) ${ }^{27}$, alors un tel jugement doit aussi s'appliquer aux poètes kurdes. Il se trouve qu'aucun de ces trois mètres, kāmil, madìd et țawīl, n'est employé plus d'une fois par un même poète. Quant au basiț, s'il est mieux représenté que les autres mètres majeurs de la tradition arabe, sa fréquence demeure faible, en persan comme en kurde, tant à l'échelle globale qu'au niveau de chaque poète. Des dix-huit poètes kurdes qui en ont fait usage, neuf ne l'ont employé qu'une fois, six deux fois, un seul trois

\footnotetext{
23. BOHAS et PAOLI 1997, p. 153.

24. AHMED et PAOLI 2010, p. 136.

25. ŠAMS-E QEYS-E RĀZī, Al-mu'̌̌am fī ma‘āyīr aš‘ār al-‘ağam, p. 108.

26. AHMED et PAOLI 2010, p. 136.

27. ṬŪsī, Me'yār al-aš'cār dar'elm-e 'arūḍ va qavāfī, p. 194.
} 
fois et trois autres quatre fois. Encore faut-il avoir à l'esprit que, chez ceux-là mêmes qui l'ont employé plus de deux fois, le basit couvre à peine plus de $2 \%$ de leur production poétique ${ }^{28}$. En définitive, les poètes Kurdes n'ont que rarement été tentés par l'emploi de mètres rares ou inusités en persan. C'est en effet pour d'autres mètres que les Kurdes, comme les Persans, ont marqué leur prédilection.

\section{Mètres privilégiés}

Si l'on tient compte d'une occurrence isolée du țawil qu'Elwell-Sutton n'a pas su identifier chez Rūdakī, on constate que seize mètres se disputent, à des degrés variables, les faveurs des Persans. Chez les Kurdes, ils sont quinze. Si l'on exclut les trois mètres propres à la poésie arabe, ainsi que le mustațil, non attesté dans le corpus poétique arabe ${ }^{29}$ et persan, les onze mètres restants en usage chez les Kurdes se retrouvent tous en persan. La métrique kurde, de ce point de vue, ne se démarque que fort peu de l'usage persan. En revanche, on trouve en persan des mètres non attestés en kurde : le muqtadab, le qarīb et le mušākil ${ }^{30}$.

Contrairement au sort qui leur est réservé en arabe, le hazağ et le ramal sont indiscutablement les champions des poésies kurde et persane. Rare en arabe ${ }^{31}$, le hazağ est le mètre de prédilection des Kurdes et des premiers poètes persans ${ }^{32}$. Sur la durée, en revanche, il perd son rang au profit du ramal. Son importance demeure toutefois incontestable, hazağ et ramal se partageant à eux deux près de la moitié des occurrences recensées en persan (49,6\%). En kurde, la popularité du duo apparaît plus significative encore : toujours en tête des mètres privilégiés, ils s'arrogent plus des quatre cinquièmes du corpus poétique $(85,1 \%)$.

Étendons notre champ d'investigation aux onze mètres communs aux deux langues iraniennes, à savoir le hazağ, le ramal, le muḍāric, le rağaz, le mutaqārib, le hafíf, le muğtatt, le sarī , le munsarih, le mutadārak et le madīd. Ensemble, ils totalisent 96,4 \% du corpus persan ; tendance accrue dans la poésie kurde, où ils s'élèvent à 99,1\%. Le constat est éloquent : les mètres les plus fréquents en persan constituent la quasi-totalité des mètres en usage en kurde.

La parenté du kurde et du persan ne se limite pas aux types de mètres privilégiés. Comme nous allons le voir, elle se confirme à l'échelle inférieure des constituants du vers : les pieds.

28. Pirtew Hekarî : 4 poèmes (2,2 \%); Nalbend : 4 poèmes $(0,6 \%)$; Mehwî : 3 poèmes $(1,4 \%)$. Voir GERDî 2003, p. 762-764.

29. PAOLI 2008, p. 145.

30. Le qarīb et le mušăkil sont considérés, avec le garīb, comme des mètres purement persans. Voir ELWELL-SUTTON 1976, p. 42. Il n'y a pas lieu de s'étonner que ces mètres ne soient pas attestés en kurde. Leur très faible fréquence en persan joua d'ailleurs certainement en leur défaveur auprès des poètes kurdes.

31. Paoli (2008, p. 364) dit du hazağ qu'il s'agit « d'une manière générale, d'un mètre extrêmement rare » dans la poésie arabe ancienne.

32. Ahmed et PAoli 2010, p. 136. 


\section{Réalisation octopartite des mètres}

Sur l'ensemble des seize mètres halīliens, la plupart des modèles de vers se présentent en arabe sous forme quadripartite ( $m u r a b b a^{c}$ ) ou sexpartite (musaddas). Dans le premier cas, le vers se fonde sur quatre pieds constitutifs (deux par hémistiche); dans le second, sur six (trois par hémistiche). Ainsi, des mètres hazağ, muḍāri`, muğtatt et muqtaḍab, la poésie arabe traditionnelle ne connaît de réalisations que quadripartites; quant au sarī', au munsarih et au madìd, ils n'apparaissent que dans leur forme sexpartite. Des mètres wäfir, rağaz, kāmil, ramal et hafif, on trouve aussi bien des exemples quadripartites que sexpartites, mais jamais octopartites (mutamman). Seuls les quatre mètres restant, à savoir le țawīl, le mutaqārib, le basit et le mutadārak, sont attestés en arabe dans leur forme octopartite ${ }^{33}$. Autrement dit, des seize mètres que compte la poésie arabe traditionnelle, douze ne sont jamais réalisés comme octopartites.

En persan et en kurde, en revanche, le constat est strictement inverse. Ainsi, si le hazağ de base, par exemple, se compose en arabe de deux pieds mafācīlun par hémistiche $(\cup---/ \cup---)$, le même mètre en persan ou en kurde se voit augmenté du double, avec quatre mafấîlun par hémistiche $(\cup---/ \cup---/ \cup---/ \cup---)$. De fait, plus de $74 \%$ de la production poétique kurde et près de $76 \%$ du corpus persan recensé s'avèrent constitués de réalisations octopartites. Il est à noter qu'en persan, le phénomène est aussi ancien que les premiers vers attestés : plus de $57 \%$ des occurrences recensées parmi les premiers poètes persans sont octopartites ${ }^{34}$. Cette conjonction de tendances met encore en évidence d'autres ressemblances frappantes entre les usages métriques en persan et en kurde.

Un autre point commun entre les traditions kurde et persane réside ainsi dans la distribution des modèles de vers: la préférence tendancielle de l'octopartite concerne dans ces deux langues des mètres différents de l'usage arabe. Pour le hazağ, les occurrences d'octopartites s'élèvent à $60 \%$ en persan et à près de $67 \%$ en kurde ; ces chiffres s'élèvent à $97 \%$ en persan et $96 \%$ en kurde pour le mudāric, et même à $100 \%$ des occurrences dans les deux langues pour le muğtatt. Une tendance similaire marque l'usage du mutaqārib et du munsarih. La réalisation octopartite des deux mètres frôle les $100 \%$ en persan; en kurde, elle est exclusive. Le parallèle se poursuit si l'on examine le ramal, le basit et le rağaz. Le premier enregistre dans les deux langues un taux de réalisation octopartite quasi identique, avec $84 \%$ en persan et $86 \%$ en kurde. Pour les deux derniers, l'usage octopartite reste majoritaire dans les deux langues iraniennes, même si les proportions diffèrent. Exclusivement octopartite en persan, le basiṭ se caractérise en kurde par un usage octopartite à près de $73 \%$. De même, le rağaz octopartite dépasse les $99 \%$ en persan et s'élève à $51 \%$ en kurde.

33. Voir la liste des modèles de vers arabes établie par BoHAs et PAOLI 1997, p. 19.

34. AhMED et PAOLi 2010, p. 136. 
Symétriquement, les mètres employés en forme sexpartite sont les mêmes en persan et en kurde. C'est le cas du hafif et du saríc - les seuls mètres susceptibles d'un usage comparable en arabe et en persan. En persan, 99,5\% des réalisations du hafif et $100 \%$ des réalisations du sarī sont sexpartites. La métrique kurde se conforme à ces usages, avec 100 \% de réalisation en sexpartite pour les deux mètres. Outre le hafiff et le sarīc, le hazağ et le ramal forment le duo le plus touché par l'usage en sexpartite. Près de $16 \%$ des ramal en persan et plus de $12 \%$ en kurde sont sexpartites. Au-delà, près de $40 \%$ des hazağs en persan et plus de $28 \%$ en kurde sont sexpartites. La convergence des tendances touche également le taux général de sexpartites dans les deux langues. La réalisation des mètres en sexpartite s'élève en persan à près de $24 \%$; en kurde à près de $20 \%$.

La réalisation quadripartite marque cependant un taux non négligeable de $5 \%$ en kurde, contre 0,04\% seulement en persan. Dans les faits, nombre de ces occurrences correspondent à des formes strophiques. Il s'agit de musammat murabbac, dont chaque strophe se compose de quatre hémistiches bipartites, les trois premiers rimant ensemble indépendamment du quatrième. Le quatrième hémistiche relie les strophes les unes aux autres par la rime : a a a b/c c c b/d d d b, etc. Or, selon Šams-e Qeys, rien n'interdit de considérer ces hémistiches bipartites comme les constituant de vers octopartites, rimés ${ }^{35}$ comme dans une qit ${ }^{c}{ }^{36}$. Dans ce cas de figure, les trois premiers demi-hémistiches du vers doivent être considérés comme musağğac (autrement dit, pourvus d'une rime interne). Si l'on appliquait cette lecture à tous les musammaț murabba' du kurde, le taux de réalisation des mètres en quadripartite diminuerait considérablement au profit des octopartites.

Par-delà la distribution des pieds dans le vers, les affinités d'usage entre métriques quantitatives kurde et persane s'étendent encore aux altérations qui les affectent.

\section{Le statut des zih̄āfāt}

La métrique halīlienne pose des mètres de base, mais aussi des variantes susceptibles d'affecter la réalisation des vers en poésie. Ces variantes, qui recensent les transformations autorisées touchant les pieds constitutifs des vers, donnent lieu dans la théorietraditionnelle à des recensions sophistiquées et à une nomenclature abondante. On distingue ainsi à chaque fois l'opération appliquée (ajout, conversion, suppression) et la position dans le pied concerné (début, milieu ou fin de pied). Les variantes se laissent répartir en deux catégories générales : les ziḥ̂āāt (sing. ziḥ̂âa) et les 'ilal (sing. 'illa).

Dans la métrique arabe traditionnelle, zihâfa désigne un ensemble de règles de transformation qui affectent les pieds internes (hašw) du vers. Il peut arriver par exemple

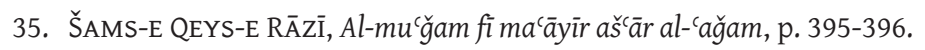

36. Voir la définition du musammaț murabbac et de la qiț $a$ dans : ŠAMĪsĀ 2008, p. 297-302.
} 
qu'un vers de hazağ, mètre fondé sur la répétition du pied mafācilun $(\cup---)$, présente dans un pied interne la variante mafāi îlu $(\cup--\cup)$, avec la dernière syllabe réalisée brève. Ce pied est alors dit affecté d'une transformation de type kaff, et nommé pour cette raison makfüf ${ }^{37}$. La 'illa, en revanche, affecte exclusivement le dernier pied de l'hémistiche ('arüd) et du vers (darb), et ne peut consister qu'en effacement ou en ajout.

Outre les positions qu'elles affectent, zihăfa et 'illa se distinguent encore en arabe par deux traits notables. Dans le poème arabe traditionnel, en effet, l'application de la zihăfa est libre, tandis que la 'illa est toujours fixe. Autrement dit, si le premier vers d'un poème est affecté d'une cilla, tous les autres vers du poème devront l'être aussi. En revanche, l'application d'une zihăfa dans un vers n'engage pas les autres vers du poème. Enfin, si la cilla peut toucher n'importe quelle syllabe du pied, la zihăfa, quant à elle, n'atteint jamais le noyau dissyllabique du pied de base, le watid ${ }^{38}$.

De ces variantes, il se trouve que les Persans ont développé une interprétation bien différente. Ainsi, les zihăāāt persanes ont un statut fixe et contraignant qui les apparente bien plutôt à la catégorie des 'ilal arabes : ils s'appliquent à l'identique à tous les vers du poème. Partant, les métriciens persans recourent au terme zihăfa dans un sens générique, pour toute variante métrique, indifféremment à la nature de la transformation et à la position du pied affectée ${ }^{39}$. Une conséquence en est que la notion de cilla est pour ainsi dire absente de la terminologie métrique persane ${ }^{40}$. Un exemple : si l'on a en persan un hazağ musaddas makfüf mahzūuf, le schéma du vers altéré ( $\cup--\cup / \cup--\cup / \cup--)$ servira de modèle fixe pour tous les hémistiches du poème. Dans cette configuration, le had qui affecte uniquement le dernier pied de l'hémistiche sera dit zihăafa au même titre que le kaff, qui s'applique aux pieds précédents ${ }^{41}$. Tous les vers étant porteurs des mêmes zihăāāt, la possibilité d'alterner les variantes au sein d'un même poème s'en trouve réduite d'autant ${ }^{42}$. Il en résulte qu'en persan, contrairement à l'arabe, les vers dérivés tendent à s'ériger en modèles de vers à part entière ${ }^{43}$.

Par son traitement des variantes, la métrique kurde s'écarte de la tradition arabe pour rejoindre le modèle persan. En kurde, toute transformation appliquée au premier vers doit s'appliquer à l'identique dans tous les vers du poème. Les deux langues iraniennes admettent

37. La métrique classique définit le kaff comme l'effacement de la septième consonne muette du pied. Voir BoHAS et PAOLI 1997, p. 157.

38. PAOLI 2008, p. 99-109; BOHAS et PAOLI 1997, p. 157-158.

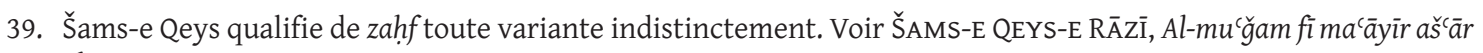
al-'ağam, p. 73-74.

40. Il s'agit d'une anomalie de la métrique persane traditionnelle, le statut fixe et obligatoire des variantes au sein du poème les apparentant plutôt aux 'ilal tels que les définit la théorie de base.

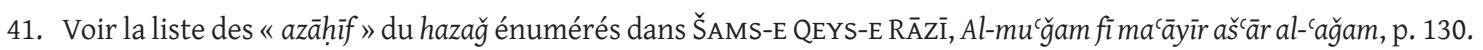

42. D’où le nombre très élevé de modèles de vers recensés en persan, relativement à l'arabe. D'après Nağafî, on trouverait en persan près de 400 modèles de vers. Voir NAĞAFİ 2008, p. 25.

43. UTAS 1994, p. 133. 
toutefois quelques dérogations à cette règle ; ces exceptions ne comptent plus, alors, au nombre des variantes (ziḥâāat). Elles relèvent en propre des licences poétiques (ehtiyārāt-e $\check{s} \bar{a}^{c}$ erì). Nous nous contenterons d'en donner ici deux exemples. La première concerne le pied initial de l'hémistiche; si le pied débute par deux syllabes brèves, une licence autorise la libre réalisation, en brève ou en longue, de la syllabe initiale. Ainsi, dans un ramal affecté $\mathrm{du}$ habn ${ }^{44}$ par exemple, le pied de base fācilātun $(-\cup--)$ est réalisé facilātun $(\cup \cup--)$, comme dans le ramal musaddas mahbūn ( $\cup \cup--/ \cup \cup--/ \cup \cup--)$. Cependant, une licence autorise le poète à réaliser le pied initial de l'hémistiche dans sa forme de base, avec une syllabe initiale longue $(-\cup--M \cup--/ \cup \cup--)$. Une seconde licence autorise le poète à remplacer deux syllabes brèves successives par une syllabe longue, sauf en position initiale ou finale d'hémistiche ${ }^{45}$. Ainsi, dans notre exemple de ramal, la synérèse est possible aussi bien dans le deuxième et dans le troisième pied. Dans un poème sur ce modèle, il se peut donc que l'on rencontre un hémistiche du type $(-\cup--/---/---)$, réalisation parfaitement tolérée à condition de ne pas s'appliquer à tous les vers du poème. Il se trouve que ces deux licences poétiques, fort répandues en persan, sont également fréquentes en kurde ${ }^{46}$.

L'intimité des métriques kurde et persane est de surcroît entérinée par une transformation particulière : le harb.

\section{Le pied ahrab}

Parmi les variantes recensées dans la métrique classique, certaines résultent de la combinaison de deux transformations simples. On les appelle, pour cette raison, « complexes » (murakkab). L'effacement de la syllabe brève du watid mağmū̄c $(\cup-)$ à l'initiale de certains mètres est dit harm. Nous avons déjà évoqué plus haut l'altération nommée kaff. Il est des cas, dans les mètres hazağ et muḍ̄aric en particulier, où ces deux transformations se combinent dans un même pied. Cette opération complexe est appelée harb, et le pied affecté, ahrab ${ }^{47}$.

Fondés sur la base des données livrées par Elwell-Sutton et Gerdî, les tableaux III et IV ci-contre font état des mètres susceptibles d'être réalisés en ahrab, avec le nombre de poèmes recensés et les modèles de vers attestés. Les pourcentages évaluent le ratio de réalisations en ahrab pour le mètre donné.

44. Dans la poésie classique, habn désigne l'effacement de la deuxième consonne muette du pied. Voir BoHAs et PAOLI 1997, p. 156.

45. NAǦAFī 2015, p. 26-32.

46. Voir l'usage de ces licences dans les modèles de ramal mahbūn et hafîf mahbūn en persan et en kurde chez HुĀNLARĨ 2007, p. 265-266; ŠAMĪSĀ 1997, p. 45-47 ; GERDî 1999, p. 160-170, 176-183.

47. PAOLI 2008, p. 100-103. 


\begin{tabular}{|l|c|c|c|}
\hline mètres & nb. de modèles de vers & nb. de modèles ahrab & $\%$ \\
\hline hazağ & 18 & 9 & 50 \\
mudāāic & 8 & 5 & 62 \\
\hline qarīb & 4 & 2 & 50 \\
mètre & nb. de poèmes & nb. de poèmes en ahrab & $\%$ \\
\hline hazağ & 4465 & 2239 & 50,1 \\
muḍāric & 3459 & 3155 & 99,8 \\
\hline qarīb & 58 & 47 & 81 \\
\hline
\end{tabular}

Tableau III

\begin{tabular}{|l|c|c|c|}
\hline mètres & nb. de modèles de vers & nb. de modèles ahrab & $\%$ \\
\hline hazağ & 22 & 12 & 54 \\
\hline mud̦āric & 6 & 5 & 83 \\
\hline mètre & nb. de poèmes & nb. de poèmes en ahrab & $\%$ \\
\hline hazağ & 4287 & 1103 & 25,7 \\
\hline muḍāric & 468 & 467 & 99,7 \\
\hline
\end{tabular}

Tableau IV

En persan, trois mètres sont susceptibles d'être affectés par le harb : le hazağ, le muḍāric et le qarib, les seuls à présenter dans leur formule de base un pied quadrisyllabe à watid $m a g ̆ m u$ ' initial $([\cup-]--)$. De ces trois mètres, seuls les deux premiers sont en usage en kurde. Comme il apparaît dans ces tableaux, les poètes dans les deux langues ont fait un usage abondant du harb, puisqu'il affecte au moins la moitié des modèles de vers attestés pour ces mètres. Le phénomène est d'autant plus frappant si l'on observe le nombre de poèmes composés sur ces mètres. On constate alors une prédominance spectaculaire des modèles ahrab pour le muḍāric dans les deux langues. Quant aux poèmes en hazağ ahrab, ils sont également très bien représentés, quoique l'on observe un écart important entre les deux corpus, les Persans ayant davantage développé ces modèles que les Kurdes.

On note encore un autre point de convergence entre l'usage kurde et persan des modèles ahrab, par quoi ils se distinguent de la pratique arabe. En arabe, le caractère aléatoire des zihhăfät s'applique au harm, qui n'apparaît le plus souvent que dans le premier vers du poème. En d'autres termes, un poème arabe en hazağ ou en muḍāric affecté d'un harb conserve malgré tout sa structure générale, avec un premier pied à watid mağmu $\bar{u}^{c}$ initial ([ $\cup-]--)^{48}$. En persan et en kurde, à l'inverse, on ne pourra pas rencontrer un tel pied dans un modèle affecté du harb. Ainsi, dans les deux langues iraniennes, la formule du hazağ mutamman ahrab est $(--\cup / \cup---/--\cup / \cup---)$, et celle du mudāaric mutatamman 


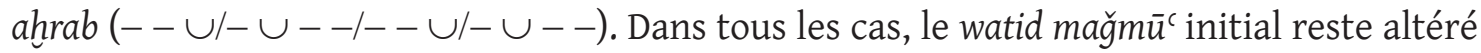
dans tous les hémistiches du poème. Ce phénomène n'a pas dissuadé les métriciens persans de recenser sous le nom de hazağ et de muḍāric les modèles de vers correspondants, ni les Kurdes de leur emboîter le pas ${ }^{49}$. Pourtant, si l'on reconnaît le rôle définitoire du watid dans la classification des mètres, l'absence de watid mağmū' initial serait de nature à remettre en cause la pertinence de cette dénomination. En persistant à les apparenter au hazağ et au mudāric', les métriciens, persans et kurdes, ont privilégié ici l'analyse la plus cohérente avec le système métrique de leur langue ${ }^{50}$.

\section{Conclusion}

S'il n'est nulle part fait mention des langues arabe et kurde dans l'introduction du Nûbar, cela n'a rien d'une négligence. Certes, l'enseignement prodigué dans les maktabs puise très largement aux sources arabes. En matière littéraire, cependant, c'est surtout aux Persans que les Kurdes sont redevables ${ }^{51}$. Ehmed Xanî en est bien conscient, lui qui loue, en introduction à son manuel, le Prophète que « les Arabes, les 'Ecem (Persans), les Kurdes et les Rûm $\hat{\imath}$ (Turcs) suivirent ${ }^{52}$ ». De semblables mentions des peuples "dominants » se retrouvent ailleurs dans son œuvre poétique. Dans son célèbre masnawī d'amour, l'auteur s'interroge ainsi sur le statut du peuple kurde, dominé par ses puissants voisins :

Mehkûm 'eleyhî û se'alîk

Emmaji ezel Xudê wesa kir
Mexlûb û mutî‘̂ Turk $\hat{u}$ Tacîk 53

Ef Rûm û 'Ecem li ser me rakir ${ }^{54}$

Condamnés à la misère, [Les Kurdes sont] dominés et asservis aux Turcs et aux Persans.

Or Dieu le voulut ainsi de toute éternité, lui qui nous imposa ces Turcs et Persans.

49. Pour une attestation ancienne de cette appellation en persan, voir ŠAMS-E QEYS-E RĀZĪ, Al-mư̌ğam fi ma‘āyìr ašăar al-'ağam, p. 136. En kurde, la plus ancienne attestation se rencontre, précisément, dans le Nûbar de Xanî. Les deux modèles de vers donnés ici en exemple sont respectivement illustrés dans le $9 e$ et le 6e qit' $a$ de son manuel. L'auteur les désigne du nom de muḍāric ahrab et de hazağ ahrab. Voir XANî, Nûbar, p. 13 et 21 (signalons au passage une erreur de lecture commise par l'éditeur, qui transcrit ahzab (ehzeb, dans sa transcription en caractères latins) au lieu d'ahrab. Il s'agit sans doute d'une mauvaise lecture des lettres pointées, le point sur la lettre $/ \mathrm{h} /$ ayant été reportée, à tort, sur la lettre suivante, $/ \mathrm{r} /$, produisant la lecture $/ \mathrm{h} /$ et $/ \mathrm{z} /$. Or, le terme ahzab est inconnu du lexique des variantes métriques. Voir XANî, Nûbar, p. 18 et 25 ; ŠAMİsā 1996, p. 16-17).

50. AHMED et PAOLI 2010, p. 139-140.

51. Dans Mem û Zîn, Xanî affiche à plusieurs reprises sa familiarité avec la littérature persane. Il évoque notamment les personnages des masnawìs d'amour légendaires Xosrow-o Šìīin, Leylī-o Mağnūn, Yūsof-o Zoleyxāa, Vìs-o Rāminn et Vāmeq-o 'Ožrā, et mentionne Neẓāmī et Ğāmī, auteurs des trois premiers. Xanî ambitionne alors de situer son ouvrage à rang égal avec ces chefs-d'œuvre. Voir XANî, Mem û Zîn, p. 19, 51; NEẒ̄ĀMī GANǦAVİ, Xosrow-o Šìin ;

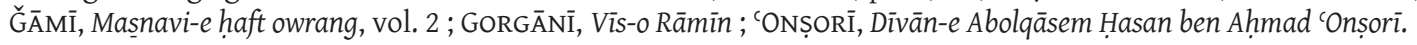

52. Les termes 'Ecem ('Ağam) et Rûmî (Rūmì) désignent alors également les Persans et les Turcs. Le parallèle entre les synonymes d'un distique au suivant est révélateur.

53. Tacîk (Tāğìk) désigne en l'occurrence le peuple persanophone : les Persans. Voir ‘AMīD 1990, p. 523.

54. XaNî, Mem û Zîn, p. 43. 
Dans le préambule de Mem û Zîn, la mention des Turcs et des Persans reparaît encore à trois reprises, chaque fois dans le même ordre ${ }^{55}$, à l'exception de la dernière ${ }^{56}$. Cet ordre reflète alors une réalité politique. L'ouest du Kurdistan, dont est originaire Xanî, étant sous domination ottomane, les Turcs viennent en premier; viennent ensuite les Persans, qui, sous les traits de l'empire safavide, règnent à la même époque sur l'est du Kurdistan ${ }^{57}$.

Dans l'introduction du Nûbar, cependant, c'est de la circulation du savoir dont il est question; la liste des peuples se fait alors reflet de la genèse du savoir et de l'ordre de la transmission. Là, les Arabes viennent en premier, car c'est en arabe que le Coran a été révélé au Prophète, premier représentant de l'Islam et premier d'entre les maîtres du savoir religieux. C'est encore de l'arabe que procèdent les arts du discours, la métrique ('arūd) et l'éloquence (balāga). Viennent ensuite les Persans, qui, relais entre les Arabes et les Kurdes, assurèrent véritablement la transmission des savoirs poétiques et des techniques littéraires. La métrique quantitative, nous venons de le voir, en offre peut-être la preuve la plus éclatante - le Nûbar lui est consacré, tout autant qu'au lexique. L'hommage au Persan, tantôt inspirateur et tantôt oppresseur, est parfois explicite dans l'œuvre de Xanî. La dette qui lui est due s'exprime, dans l'introduction au Nûbar, par le silence imposé aux autres langues. Nommer l'arabe, nommer le kurde, c'eût été faire l'économie du passeur persan, c'eût été méconnaître le rôle du chaînon manquant. En composant ce tout premier manuel de 'arū

55. XANî, Mem û Zîn, p. 45, 47.

56. L'ordre des peuples, alors, est inversé, car il ne s'agit plus de désigner un fait réel, mais d'évoquer un possible rêvé : si les Kurdes étaient unis, explique Xanî, ils pourraient à leur tour dominer « les Turcs, les Arabes et les Persans ». Voir XANî, Mem û Zîn, p. 47.

57. XeZnedar 2002, p. 344. 


\section{Bibliographie}

\section{Sources}

\section{Euvres poétiques}

AXTEP̂̂, Şêx Evdirehman, 2013, Dîwana Rûhî, éd. Rênas JIYAN, Diyarbakir, Belkî.

AXTEPÎ, Şêx Hesîb, 2009, Dîwana Hesbî, Istanbul, DOZ.

BADî, Isma'îl, 2011, Remezanê Cizîrî, di dwîfçûn û twîjandina dîtir da qeftek ji helbestên wî, Duhok, Senterê Vekûlînên Kurdî û Parastina Belgenaman.

BIDLîsî, Haris, 2004, Leyla û Mecnûn, éd. Tehsîn Ibrahîm DosKî, Duhok, Spîrêz.

CEGERXWîn, 2002, Agir û Pirûsk, Dîwan 1, Istanbul, Avesta.

CEGERXWÎN, 2002, Sewra Azadî, Dîwan 2, Istanbul, Avesta.

CEGERXWîn, 2002, Kîme Ez, Dîwan 3, Istanbul, Avesta.

CEGERXWÎN, 2002, Ronak, Dîwan 4, Istanbul, Avesta.

CEGERXWÎN, 2002, Zend-Avista, Dîwan 5, Istanbul, Avesta.

CEGERXWîn, 2002, Şefaq, Dîwan 6, Istanbul, Avesta.

CEGERXWîN, 2002, Hêvî, Dîwan 7, Istanbul, Avesta.

CEGERXWîn, 2002, Aşitî, Dîwan 8, Istanbul, Avesta.

CEGERXWîn, 2002, Salar û Mîdya, Dîwan 9, Istanbul, Avesta.

CEGERXWÎN, 2002, Şerefnameya Menzûm, Dîwan 10, Istanbul, Avesta.

CIzîRî, Seyîd Qedrî, 2009, Dîwana Seyîd Qedrîyê Cizîrî, éd. Tehsîn Ibrahîm Doskî, Duhok, Çapxana Xanî.

DAHÎ, Mela Nezîr Muhemmed Tahir Bedewî, 2004, Dîwana Dahî, éd. Tehsîn Ibrahîm Doskî, Duhok, Spîrêz.

Doskî Tehsîn Ibrahîm (éd.), 2010, Dûferhengên vehandî, Nûbar û Mirșād al-Ațfāl yên Ehmedê Xanîûu Muhemmed Kerbelayî, Erbil, Ekadîmiyay Kurdî.

Doskî Tehsîn Ibrahîm (éd.), 2011, Mela 'Ebdillahê Hîzanî û şa'irên malbata wî, Duhok, Spîrêz.

Doskî Tehsîn \& ŞERMEXî, Husên (éds.), 2011, Parçeyek ji dîwana Laxerî, Duhok, Spîrêz.

FINDIKî Dilhezîn, 1997, Dîwana xebatker Dilhezîn Findika Buhtan (Dilhezîn Findikî), Duhok, Çapxana Xebat.

GORAN `Ebdulla, 1980, Dîwanî Goran, Muhemmed MELA KeRîM (éd.), Bexda, Kořî Zanyarî Kurd.

GORGĀNī Fahroddīn As`ad, 1998/1377, Vīs-o Rāminn, Mohammad RowšAN (éd.), Téhéran, Ședāye Mocāṣer.

ĞĀMİ Noroddīn 'Abdolraḥmān ben Aḥmad, 1999/1378, Mașnavi-e ḥaft owrang, vol. 2 (Yūsof-o Zoleyhă, Leylīo Mağnūn va Heradnāme-ye eskandarī), A'lāhān AFȘAḤZĀD et alii (éds.), Téhéran, Daftar-e Našr-e Mīrās̄e Maktūb.

HADî, Mela Muhemmed Sibxetulla Liceyî, 2007, Dîwana Hadî, éd. Tehsîn Ibrahîm DosKî, Duhok, Spîrêz.

HEYDERî, Ehmed \& Heyderî, Muhemmed-Emîn, 2012, Dîwana Heyderî, éd. Ziyaeddîn HEYDERî, Istanbul, Nûbihar.

HEZRÛYî,, Mela 'Ebdulfettah, 2012, Dîwana Fethî, éd. Tehsîn Ibrahîm DosKî, Duhok, Spîrêz.

NEẒĀMī GANǦAVĪ, Elyās ben Yūsof, 2000/1379, Xosrow-o Šìrīn, Behrūz SַERVATīYĀN (éd.), Téhéran, Tūs. 
'ONȘORĪ, Abolqāsem, 1962/1341, Dīvān-e Abolqāsem Ḥasan ben Aḥmad 'Onșorī, Yahyā QARĪB (éd.), 2e éd., Téhéran, Ketābhāne-ye Ebn-e Sīnā.

SERDAŶ̂, Selahiddîn 'Ebdulla Wanî, 2012, Dîwana Serdayî, éd. Tehsîn Ibrahîm DosKî, Duhok, Weşanên Êketiya Nivîserên Kurd.

SIYAHPOȘ, 2011, Seyf-ul Milûk û Bedî-ul Cemal, éd. Bedirxan AMEDî, Istanbul, Nûbihar (2e éd.).

TENDÛREKî, Mela Zahir, 2007, Dîwan, Mela Abdurrahman BiçıCI (éd.), Istanbul, Nûbihar.

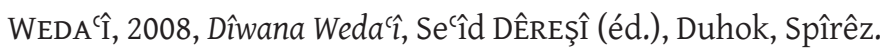

XANî, Ehmed, 1986, Nûbar, éd. Zeynelabedin KAYA, Stockholm, Weşanên Roja Nû.

XANî, Ehmed, 2008, Mem û Zîn, Tehsîn Ibrahîm Doskî (éd.), Duhok, Spîrêz.

\section{Autres ouvrages}

GoRAN 'Ebdulla, 2002, Goran, nûsîn û pexşan û wergêrawekanî, Umêd AșNA (éd.), Erbil, Dezgay Çap û Bilawkirdinewey Araz.

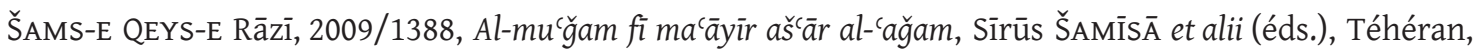
Našr-e 'Elm.

TiBRĪZī, Abū Zakariyyā Yahyā b. 'Ali al-Šaybānī (al-Hațīb), 1970, Kitāb al-kāfì fì l-‘arūd wa-l-qawāfí, éd. H.. 'A. AL-HASASĀNī̄, Le Caire, Dār al-kitāb al-'arabī li-l-țibā'a wa-l-našr.

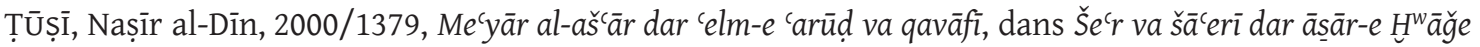
Nașir al-Dīn Ṭūsī, Mo'aẓzame EQBĀLī A'ẒAM (éd.), 2e éd., Téhéran, Sāzmān-e Čāp va Entešāāāt-e Vezārat-e Farhang va Eršād-e Eslāmī.

Études

AHMED Amr Taher \& PAOLI Bruno 2010, «La métrique des premiers vers persans », Bulletin d'Études Orientales 59, p. 127-140.

AHMED Amr Taher 2012, «La poésie de Goran, d'Est en Ouest », Études kurdes 11, p. 65-80.

'AMīd Ḥasan 1990/1369 (7éd.), Farhang-e färsī-e 'amìd, Téhéran, Mo’assese-ye Entešārāt-e Amīr Kabīr.

'ARIF Muhemmed Muhemmed-Nûrî 1974, « Te’sîrî ziman û edebî farsî le ser edebî kurdî », Govarî Kolêcî Edebiyat 18, p. 72-85.

BÎMAR 'Ebdulrezzaq 1970, « Danîştinêk legeĺ Goranda », Beyan 2/2-3, p. 30.

BîMAR 'Ebdulrezzaq 1992, Kêş û mosîqay heĺlbestî kurdî, Bexda, Dezgay Roşinbîrî û Bilawkirdinewey Kurdî.

BoHAS Georges \& PAOLI Bruno 1997, Aspects formels de la poésie arabe, Toulouse, AMAM.

BOREKEYî Sidîq (Sefîzade) 2008, Mêjûy wêjey kurdî, vol. 1, Erbil, Dezgay Çap û Bilawkirdinewey Aras.

ELWell-SutTon Laurence Paul 1976, The Persian Metres, Cambridge, Cambridge University Press.

GERDî 'Ezîz 1999, Kêşî şếrî kilasîkî kurdî û berawird kirdinî legel 'erûzî 'erebî û kêşî şếrî farsîda (lêkolîneweyekî şîkarî berawird e), Erbil, Wezaretî Roşinbîrî.

GERDî 'Ezîz 2003, Raberî kêşî șếrî kilasîkî kurdî, Erbil, Wezaretî Roşinbîrî.

HEMEXAN Nûrî Faris 2004, 'Erûzî kurdî, Erbil, Dezgay Çap û Biĺawkirdinewey Araz.

HARDĪ Aḥmad 2004, Al-`arūẹ fí al-ší'r al-kurdī, Erbil, Manšūrāt al-Mağma' al-'Tlmī al-Kurdistānī.

HōĀNLARĪ Parvīz Nātel 2007/1386 (7e éd.), Vazn-e še`r-e fārsī, Téhéran, Entešārāt-e Tūs. 
NAĞAFĩ Abolḥasan 2008/1387, " ‘Arūḍ-e qadīm dar barābar-e 'arūḍ-e ğadīd », in Ḥasan QARĪBİ (éd.), Nahostīn mağmū'e sohanrānī-hā-ye moštarak-e Farhangestān-e zabān va adab-e färsī va Bonyād-e Irān-šenāsī, Tehrān, Entešārāt-e Ferhangestān, p. 3-47.

NAǦAFì Abolhasan 2015/1394, Ehtiyārāt-e ša'erī va maqālehā-ye digar dar 'arūḍ-e fārsī, Téhéran, Entešārāt-e Nīlūfar.

PAOLI Bruno 2008, De la théorie à l'usage. Essai de reconstitution du système de la métrique arabe ancienne, Damas, Institut français du Proche-Orient.

ŠAmīsā Sīrūs 1996/1375 (3e éd.), Farhang-e 'arūdī, Téhéran, Našr-e Ferdows.

ŠAMīsĀ Sīrūs 1997/1376 (13e éd.), Āšnāyī bā carūḍ va qāfiye, Téhéran, Našr-e Ferdows.

ŠAMīsĀ Sīrūs 2008/1387 ( $3^{e}$ éd.), Anvā'ce adabī, Téhéran, Našr-e Mìtra.

UTAS Bo 1994, "Arabian and Iranian elements in New Persian prosody», dans L.JoHANSon \& B. UTAS (éd.), Arabic Prosody and its Applications in Muslim Poetry, Istanbul-Stockholm, Swedish Research Institute in Istanbul (Transactions, vol. 5), p. 129-141.

VALI Shahab 2011, Les Yârsâns. Aspects mythologiques - Aspects doctrinaux, Éditions Universitaires Européennes.

VALI Shahab 2012, «La littérature religieuse des Kurdes Yarsan », Études kurdes 11, p. 57-64.

XEZNEDAR Marif 1962, Kêş û qafiye le şếrî kurdîda, Bexda, Çapxaney el-Wefa.

XEZNEDAR Marif 2001, Mêjûy edebî kurdî, vol. 1, Erbil, Dezgay Çap û bilawkirdinewey Aras.

XEZnedAr Marif 2002, Mêjûy edebî kurdî, vol. 2, Erbil, Dezgay Çap û bilawkirdinewey Aras. 